\title{
Optimization of advanced manufacturing processes using socio inspired cohort intelligence algorithm
}

\author{
Ishaan R. Kale* (D), Mayur A. Pachpande, Swapnil P. Naikwadi(D), and Mayur N. Narkhede(D) \\ Symbiosis Institute of Technology, Symbiosis International (Deemed University), Near Lupin Research Park, Gram: Lavale, Tal. \\ Mulshi, Pune, Maharashtra 412115, India
}

Received: 14 June 2021 / Accepted: 28 October 2021

\begin{abstract}
The demand of Advanced Machining Processes (AMP) is continuously increasing owing to the technological advancement. The problems based on AMP are complex in nature as it consisted of parameters which are interdependent. These problems also consisted of linear and nonlinear constraints. This makes the problem complex which may not be solved using traditional optimization techniques. The optimization of process parameters is indispensable to use AMP's at its aptness and to make it economical to use. This paper states the optimization of process parameters of Ultrasonic machining (USM) and Abrasive water jet machining (AWJM) processes to maximize the Material Removal Rate (MRR) using a socio inspired Cohort Intelligent (CI) algorithm. The constraints involved with these problems are handled using static penalty function approach. The solutions are compared with other contemporary techniques such as Particle Swarm Optimization (PSO), Artificial Bee Colony (ABC), Modified Harmony Search (HS_M) and Genetic Algorithm (GA).
\end{abstract}

Keywords: Advanced machining processes / complex problems / linear and nonlinear constraints / cohort intelligence algorithm

\section{Introduction}

Traditional manufacturing processes were very useful in early decades. As the world started demanding more in terms of standards and quality, it is very difficult to manufacture certain products due to its complex shape, size and quantity as it is beyond the limit of traditional manufacturing processes. Therefore, industrial sector has modified traditional manufacturing processes named as Advance Manufacturing Processes (AMP).

The real-world applications of AMPs such as Light Amplification Stimulated Emission of Radiation machining problem $[1,2]$ which is used for cutting process. In this machine, a focused and precise laser beam is passed through the material by accomplishing cutting of material therefore other processes like wire electric discharge machining [3], electric discharge machining [3], electrochemical machining [4], electro-beam machining problem [5], ultrasonic machining [6] and more were invented for specific operations while considering human safety and standards issues. These are further classified under mechanical processes, chemical and electro chemical processes, thermal and electro thermal processes and finishing processes [7]. In these problems, precise selection

\footnotetext{
* e-mail: ishaan.kale@sitpune.edu.in
}

of process parameters plays a key role to manufacture the product in a reasonable cost and time. Parameters such as cutting speed, cutting depth, number of passes of the wheel or table, amplitude, magnitude, volume and speed of the tool are considered under various of machining processes.

Water Jet Machining (WJM) process is a nontraditional machining process in which water at high pressure and velocity is used to cut the softer materials. An application of regression modeling and Taguchi Method were used to optimize AWJM processes parameter [8]. It has shown the great potential to improve the process parameter and to obtain the required surface roughness. The application of elasto-plastic finite element analysis used to simulate the $3 \mathrm{D}$ erosion in AWJM of grade 5 Titanium alloy [9]. Li and Wang [10] presented the drilling and slotting machining processes on Ti-6Al-4V alloy using AWJM. USM problem [11] was designed to maximize the MRR, which was solved using ABC, HS_M and PSO. While [12] carry forward undetermined non-conventional optimization techniques such as Gravitational Search Algorithm (GSA) and Fireworks Algorithm (FWA) on USM processes. [13] carried out the experimentation on USM using Adaptive Neuro Fuzzy Inference System (ANFIS) and Independent Component Analysis (ICA) were related together to optimize USM for multi-responses, therefore obtained data were bought together for development of mapping relationship connecting inputs and 
ANFIS. Metaheuristic algorithm such as Cuckoo Search (CS) and Chicken Swarm Optimization (CSO) were used by [14] to solve the USM problem.

Apart from these, there are several socio-inspired optimization algorithms proposed so far, such as PC [15], SOS [16], Teaching Learning Based optimization (TLBO) [17], so on and so forth. The CI algorithm is also one of the socio inspired algorithm proposed by [18]. It models the learning behavior of the candidates such as following, interacting, cooperating and competing with every other candidate in the cohort. It was modified to solve constrained problems and applied to solve combinatorial NP-hard 0-1 Knapsack problem with the number of items varying from 4 to 75 [19]. The probability-based constraint handling techniques was used to handle the constraints. Similar approach was also applied for solving real world combinatorial problems from healthcare and logistics domains and large sized complex problems from the Cross-Border Supply Chain domain [20], Traveling Salesman Problem (TSP) [21] and several benchmark problems [18]. A self-adaptive Cohort Intelligence (SACI) algorithm [22] was proposed using tournament mutation operator and a self-adaptive scheme to update the sampling interval. It is tested on several benchmark problems and obtained promising results. The static and dynamic penalty function approach is incorporated in CI (CI-SPF and CI-DPF) for solving several test problems and manufacturing engineering problems [23]. The multi-CI [24] and variations of CI [25] were used to solve the AWJM problem for minimization of surface roughness. The CI$\mathrm{SPF}$ is adopted for solving complex problems from truss structure and mechanical engineering domain $[26,27]$. Being observed the limitation in CI-SPF, the CI is incorporated with Self Adaptive Penalty Function (SAPF) approach [28]. Further, some intrinsic properties of $\mathrm{CI}$ and Colliding Bodies Optimization (CBO) are combined to formed a new hybrid metaheuristic CI-SAPF-CBO. Using CI-SAPF and CI-SAPF-CBO, 40 problems from truss structure domain, design engineering domain, linear and nonlinear problems and real-world manufacturing domain problems [28].

In this paper, very first time a socio inspired Cohort Intelligence (CI) algorithm is validated by solving AMPs problems such as abrasive water jet machining and ultrasonic machining problems. These problems are associated with linear and nonlinear constraints which are handled using static penalty function approach. The constrained version of CI algorithm [26] is used to investigate these problems. The solutions obtained using CI algorithm are compared with other contemporary algorithms and results are discussed.

The paper is organized as follows: The CI algorithm along with its characteristics are described in Section 2. The framework of CI and its flowchart is presented in Section 2.1. Section 3 demonstrates the constraint handling SPF approach. Section 4 discussed the AMP problems, in the same section the results comparison with contemporary algorithms and its analysis is discussed. Finally, the conclusion is presented in Section 5 .

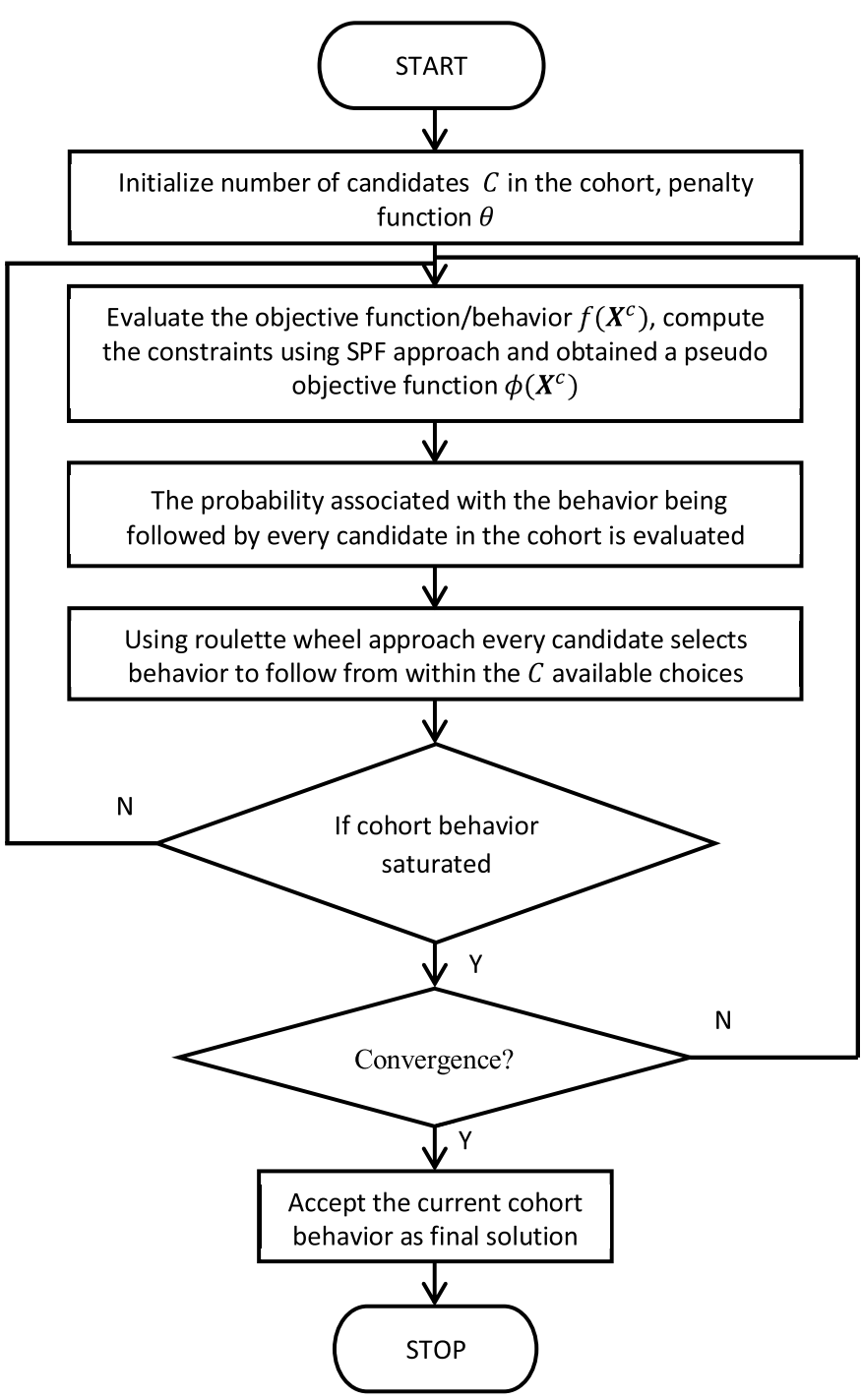

Fig. 1. Flowchart of CI algorithm.

\section{Cohort intelligence algorithm}

The CI algorithm [18] models the social tendencies of learning candidates of a cohort. Every candidate in the cohort iteratively attempts to achieve a goal which is common to all. For this, every candidate employs roulette wheel approach and selects another candidate to follow which may result in the improvement of its own behavior. This makes every candidate learn from one another and helps the overall cohort behavior to evolve. The cohort behavior could be considered saturated, if for considerable number of learning attempts the behavior of every candidate does not improve considerably and becomes almost same. The flowchart is presented in Figure 1 [26]. The characteristics of CI algorithm [28] are as follows:

- It models the learning mechanism of cohort candidates. Every candidate has inherently common goal to achieve the best behavior by improving its qualities. The interaction and competition are the two natural instincts of every cohort individual. These are achieved through 
roulette wheel selection and further sampling in the close neighborhood of the selected (being followed) candidate. For details refer to $[18,21]$.

- Every candidate observes itself and every other candidate in the cohort to improve its individual behavior and associated qualities.

- In CI algorithm, at the end of every learning attempt every candidate independently updates its search space.

- The problem with large number of variables and constraints can be efficiently handled $[20,26]$.

\subsection{Framework of $\mathrm{Cl}$}

In the context of CI candidate follows other candidate which is probabilistically chosen from the cohort using roulette wheel approach. The CI algorithm [18] is mathematically expressed as follows:

Step 1: Consider a cohort with $C$ number of candidates; every individual candidate $c(c=1,2, \ldots, C)$ contains a set of attributes/variables $\boldsymbol{X}^{c}=\left(X_{1}^{c}, X_{2}^{c}, \ldots, X_{i}^{c} \ldots, X_{n}^{c}\right)$ which makes the behaviour of an individual candidate $f\left(\boldsymbol{X}^{c}\right)$. The initial solution is randomly generated similar to the other population-based technique as follows:

$$
\boldsymbol{X}^{c}=\Psi^{\text {lower }}+\left(\Psi^{\text {upper }}-\Psi^{\text {lower }}\right) \cdot r \text { and }(1, n) . s
$$

Step 2: A static penalty function (SPF) approach is incorporated to handle the constraints and obtained pseudo objective function $\varphi\left(\boldsymbol{X}^{c}\right)$ (refer Sect. 3).

Step 3: The probability of selecting behavior $f\left(\boldsymbol{X}^{c}\right)$ of every associated candidate $c(c=1,2, \ldots, C)$ is calculated as follows:

$$
p^{c}=\frac{{ }^{1 / \phi}\left(\boldsymbol{X}^{c}\right)}{\sum_{c=1}^{C} 1 / \phi\left(\boldsymbol{X}^{c}\right)}
$$

Step 4: Every individual candidate $c(c=1,2, \ldots, C)$ generates a random number $r \varepsilon[0,1]$ and using roulette wheel approach decides to follow the corresponding behaviour and associated attributes.

Step 5: Every candidate $c(c=1,2, \ldots, C)$ shrinks the sampling interval $\Psi_{i}^{c}, i=1, \ldots, n$ associated with every variable $X_{i}^{c}, i=1, \ldots, n$ to its local neighborhood. This is done as follows:

$$
\Psi_{i}^{c} \varepsilon\left[X_{i}^{c}-\left(\Psi_{i} / 2\right), X_{i}^{c}+\left(\Psi_{i} / 2\right)\right]
$$

Where $\Psi_{i}=\Psi_{i} \times R ; R$ is sampling space reduction factor.

Each candidate $c(c=1,2, \ldots, C)$ samples their qualities from within the updated sampling interval $\Psi_{i}^{c}$ and computes the function values. This makes the cohort is available with $C$ updated behaviors represented as $F^{C}=$ $\left\{f\left(\boldsymbol{X}^{1}\right), \ldots, f\left(\boldsymbol{X}^{c}\right), \ldots, f\left(\boldsymbol{X}^{C}\right)\right\}$.

Step 6: The cohort behavior could be considered saturated, if there is no significant improvement in the behavior $f\left(\boldsymbol{X}^{c}\right)$ of every candidate.
If either of the two criteria listed below is valid, accept any of the $C$ behavior from current set of behavior in the cohort as the final objective function values as final solution and stop, else continue to Step 1.

- If maximum number of attempts exceeded.

- The cohort reaches a saturation state. There is no significant improvement in the further learning attempts.

\section{Static penalty function (SPF) approach}

In general, the constrained optimization problem is expressed as follows:

$$
\begin{gathered}
\text { Minimize } f(\boldsymbol{X})=f\left(X_{1}, X_{2}, \ldots, X_{i}, \ldots, X_{n}\right) \\
\text { Subject to } g_{i}(\boldsymbol{X}) \leq 0, \quad i=1,2, \ldots, p \\
h_{i}(\boldsymbol{X})=0, \quad i=1,2, \ldots, m \\
\Psi_{i}^{\text {lower }} \leq X_{i} \leq \Psi_{i}^{\text {upper }}
\end{gathered}
$$

An exterior Static Penalty Function (SPF) constraint handling approach was widely used [29]. It is expressed as follows:

$$
P F=\theta \times\left(\sum_{i=1}^{p} g_{i}(\boldsymbol{X})+\sum_{i=1}^{m} h_{i}(\boldsymbol{X})\right)
$$

where $\theta$ is a penalty parameter and $\left(\sum_{i=1}^{p} g_{i}(\boldsymbol{X})+\sum_{i=1}^{m} h_{i}(\boldsymbol{X})\right)$ is summation of the violated constraints. The value of $\theta$ needs to be chosen arbitrary.

For the validation of proposed CI algorithm, the problems considered here are from advanced manufacturing domain. The CI algorithm is coded in MATLAB (R2019a) and the simulations are run on Windows 10 platform using an Intel Core i5, 2.5 GHz processor speed and 8GB RAM. Furthermore, both the problems are solved 30 times. The solutions obtained from CI algorithm and comparison with other contemporary algorithms are discussed in the following sections.

\section{Advanced manufacturing processes (AMP) problems}

The CI algorithm is applied to solve AMP problems such as AWJM problem and USM problem. The objective is to maximize the MRR. The constraints are handled using a static penalty function approach. Similar problems were also solved using different techniques such as ABC, HS M and PSO [11] and GA [30] with different objective function. 


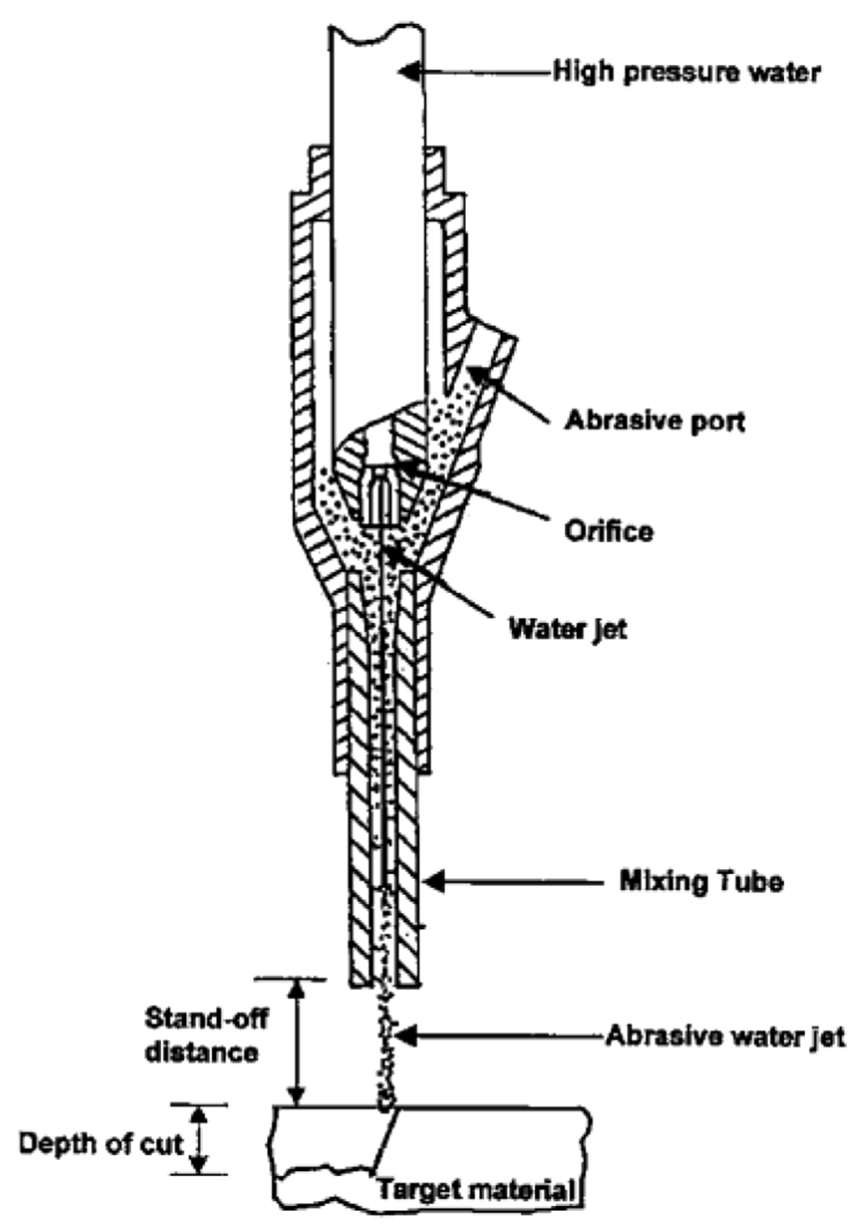

Fig. 2. Abrasive Water Jet Machining process [31].

\subsection{Problem 1: Abrasive water Jet machining (AWJM) problem}

Abrasive Water Jet machining (AWJM) process (refer Fig. 2) [32], in which velocity of water jet is increased and abrasive mixture is used to erode the workpiece. It uses the mixture of water and abrasive substance which mixed in a separate chamber and then pressurized towards nozzle for the cutting process. This process is used to cut wide variety of objects having complex dimensions. Hard materials such as metal, granite, wood, rubber, silicon, etc. are machined [32].

The AWJM problem was earlier solved using GA [33], PSO and ABC [31]. The objective is to maximize the MRR subject to power. The five decision variables considered for this model are: water jet pressure at the nozzle exit $\left(P_{w}\right)$, diameter of AWJ nozzle $d_{\text {awn }}$ feed rate of nozzle $\left(f_{n}\right)$, mass flow rate of water $\left(M_{W}\right)$ and mass flow rate of abrasives $\left(M_{a}\right)$. The constants and parameters associated to this problems are illustrated in Table 1. The problem is formulated as follows:

Maximize $M R R=\left(d_{\text {awn }} \times\left(F_{n}\left(h_{c}+h_{d}\right)\right)\right)$ where,

$$
\begin{aligned}
h_{d}= & \frac{\eta_{a} d_{a w n} \dot{M}_{a}\left[K_{1} \dot{M}_{w} P_{w^{0.5}}-\left(\dot{M}_{a}+\dot{M}_{w}\right) v_{a c}\right]^{2}}{\left(1850.8 \sigma_{f w}\right) d_{a w n^{2}} f_{n}\left(\dot{M}_{a}+\dot{M}_{w}\right)^{2}+\left(K_{1} C_{f w} \eta_{a}\right)} \\
& {\left[K_{1} \dot{M}_{w} P_{w^{0.5}}-\left(\dot{M}_{a}+\dot{M}_{w}\right) v_{a c}\right]\left(\dot{M}_{a} \dot{M}_{w} P_{w^{0.5}}\right) } \\
h_{c}= & \left(\frac{1.028 \times 10^{4.5} \zeta}{C_{k} \rho_{a^{0.4}}}\right)\left(\frac{d_{a w n^{0.2}} \dot{M}_{a}^{0.4}}{f_{n}^{0.4}}\right)\left(\frac{\dot{M}_{w} P_{w^{0.5}}}{\dot{M}_{a}}+\dot{M}_{w}\right) \\
- & \left(\frac{18.48 K_{a^{0.67}} \zeta^{\left(\frac{1}{3}\right)}}{C_{k}\left(\frac{1}{3}\right)} f_{r}^{0.4}\right)\left(\left(\frac{\dot{M}_{w} P_{w^{0.5}}}{M_{a}}+\dot{M}_{w}\right)^{\left(\frac{1}{3}\right)}\right)
\end{aligned}
$$

If $\alpha_{t} \leq \alpha_{o}$, then $h_{c}=0$

Subject to 1.0

$$
-\left(\frac{P_{w} \times M_{w}}{P_{\max }}\right) \geq 0 \text { (Surface Roughness). }
$$

The bounds for the five variables are as follows:

$$
\begin{gathered}
50 \leq P_{w} \leq 400(\mathrm{Mpa}) \\
0.2 \leq F_{n} \leq 25.0(\mathrm{~mm} / \mathrm{s}) \\
0.0003 \leq M_{a} \leq 0.08(\mathrm{~kg} / \mathrm{s}) \\
0.5 \leq d_{\text {awn }} \leq 5.0(\mathrm{~mm}) \\
0.01 \leq M_{w} \leq 0.2(\mathrm{~kg} / \mathrm{s}) .
\end{gathered}
$$

For AWJM problem, CI algorithm has obtained better solution (maximum MRR) as compared GA [34], PSO and ABC [31] (refer Tab. 2). The best, mean and worst solutions obtained form 30 trials using CI algorithm are $97.7921 \mathrm{~mm}^{3} / \mathrm{s}$, $93.1370 \mathrm{~mm}^{3} / \mathrm{s}$ and $90.9787 \mathrm{~mm}^{3} / \mathrm{s}$ with standard deviation 1.4686. The average function evaluations are 591 and average computational time is $0.81 \mathrm{~s}$. The other computational details are presented in Table 5 . The convergence plot is presented in Figure 3. It is observed that, the MRR obtained from PSO and ABC is $230.50 \mathrm{~mm}^{3} / \mathrm{s}$ and $218.49 \mathrm{~mm}^{3} / \mathrm{s}$, respectively. However, MRR for PSO and $\mathrm{ABC}$ is recalculated using the obtained decision variables by [31] where the MRR found to be $90.54 \mathrm{~mm}^{3} / \mathrm{s}$ and $88.66 \mathrm{~mm}^{3} / \mathrm{s}$, respectively. From that it is observed that CI has found better solution than GA [34], PSO and $\mathrm{ABC}[31]$.

\subsection{Ultrasonic machining (USM) problem}

The Ultrasonic Machining (USM) process (refer Fig. 4) is used in ceramics, semiconductors and glass industries. It is a material removal process which erode material in the 
Table 1. Constants used in Abrasive Water Jet Machining problem.

\begin{tabular}{lll}
\hline Constants & Details & Value \\
\hline$\rho_{a}$ & Abrasive particles Density & $3.95 \times 10^{-6} \mathrm{~kg} / \mathrm{mm}^{3}$ \\
$v_{a}$ & Velocity of abrasive particles & $0.25 \mathrm{~mm} / \mathrm{s}$ \\
$E_{y a}$ & Youngs Modulus of elasticity of abrasive particles & $350000 \mathrm{MPa}$ \\
$f_{r}$ & Roundness factor of abrasive particle & 0.35 \\
$f_{s}$ & Sphericity factor of abrasive particle & 0.78 \\
$\eta_{a}$ & Proportionality of abrasive grains effectively & 0.7 \\
\hline
\end{tabular}

Table 2. Comparison of results for solving Abrasive Water Jet Machining problem.

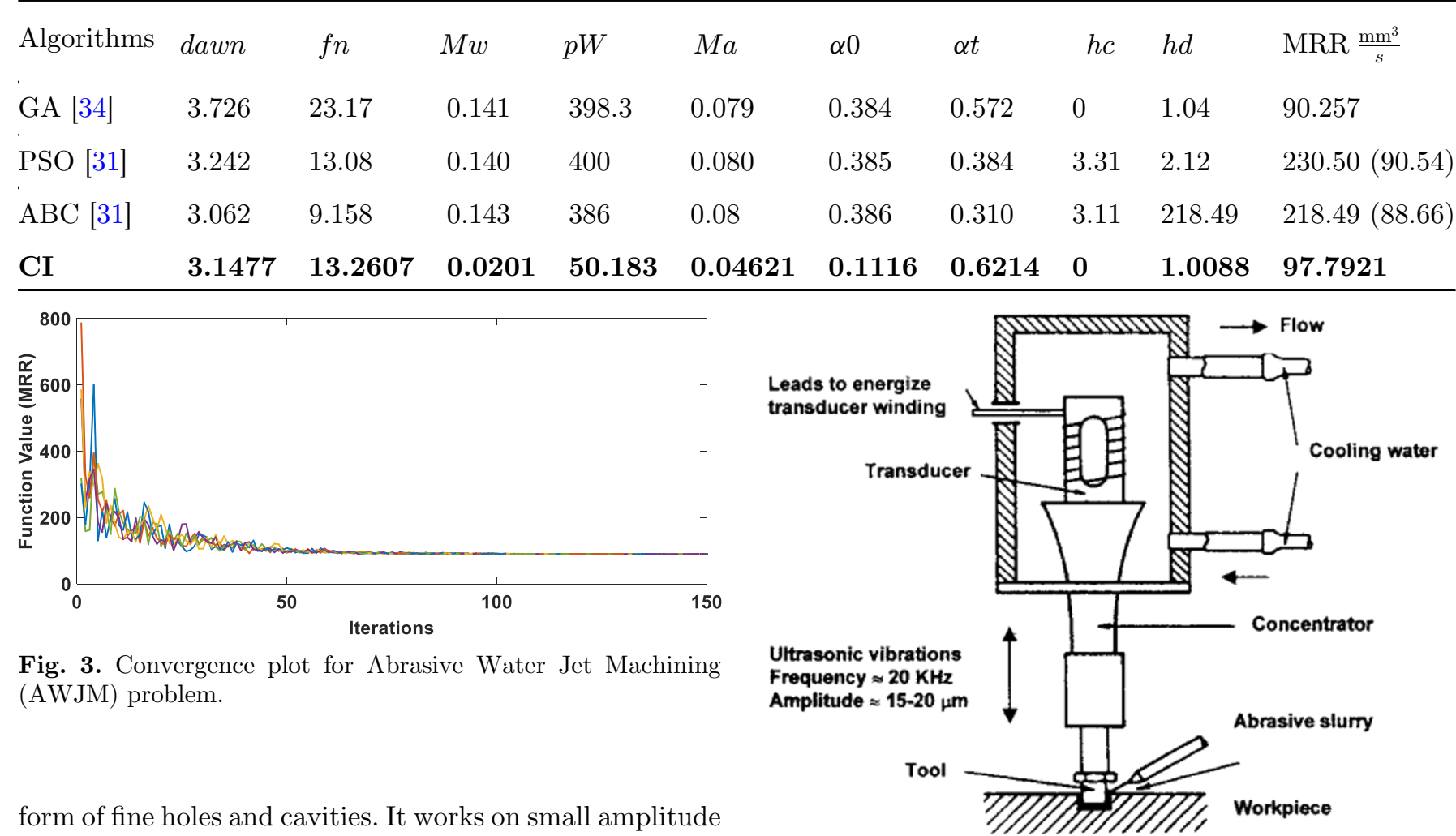
and high frequency typically in the range of 10 micro meter at $20 \mathrm{kHz}$ [35] and material removal rate (MRR) will take place in the form of fine grains by shear deformation.

The USM problem was previously solved using GA [34], PSO, ABC and HS_M [31]. The objective is to maximize the MRR. Figure $\overline{4}$ represents the USM process. The decision variables are amplitude of vibration $A_{v}(\mathrm{~mm})$; frequency of vibration $f_{v}(\mathrm{~Hz})$; mean diameter of abrasive grain $d_{m}(\mathrm{~mm})$; columetric concentration of abrasive particles in slurry $C_{a v}$, and static feed force $F_{s}(\mathrm{~N}) . K_{u}$ Is a constant of proportionality $\left(\mathrm{mm}^{-1}\right)$ relating mean diameter of abrasive grains, and diameter of projections on an abrasive grain $\left(=K_{u} d_{m}^{2}\right)$, the constant values associated with this problem is presented in Table 3

Fig. 4. Ultrasonic Machining process [31].

Maximize $M R R$

$=\left(\left(\frac{\left(4.963\left(A_{t}^{0.25}\right)\left(K_{u s m}{ }^{0.75}\right)\right)}{\left(\sigma_{f w}(1+\lambda)\right)^{0.75}}\right) \times F_{s}{ }^{0.75} A_{v}{ }^{0.75} C_{a v}{ }^{0.25} d_{m} f_{v}\right)$

$$
\begin{aligned}
& \text { Subject to } 1.0-\left(\left(\frac{1154.7}{\left(A t \times \sigma_{f w}\left(1+t_{0} \lambda\right)\right)^{0.5} \times\left(R_{a}\right)_{\max }}\right)\right. \\
& \left.\times\left(\frac{F_{s} \times A_{v} \times d_{m}}{C_{a v}}\right)\right) \geq 0
\end{aligned}
$$


The bounds for the five variables are as follows:

$$
\begin{gathered}
0.005 \leq A_{v} \leq 0.1(\mathrm{~mm}) \\
0.007 \leq d_{m} \leq 0.15(\mathrm{~mm}) \\
4.5 \leq F_{s} \leq 45.0(\mathrm{~N}) \\
10,000 \leq f_{v} \leq 40,000(\mathrm{~Hz}) \\
0.05 \leq C_{a v} \leq 0.5 .
\end{gathered}
$$

Table 3. Constant value used in Ultrasonic Machining problem.

\begin{tabular}{lll}
\hline Constant & Details & Value \\
\hline$A_{t}$ & Cutting tools Cross sectional & $20 \mathrm{~mm}^{2}$ \\
$\sigma_{f w}$ & Abrasive particle Flow stress & $6900 \mathrm{MPa}$ \\
$K_{u s m}$ & $\begin{array}{l}\text { Constant of proportionality } \\
\text { relating mean diameter of } \\
\text { abrasive grains and diameter } \\
\text { of projection on an abrasive grain } \\
R_{\max }\end{array}$ & $0.1 \mathrm{~mm}^{-1}$ \\
\hline
\end{tabular}

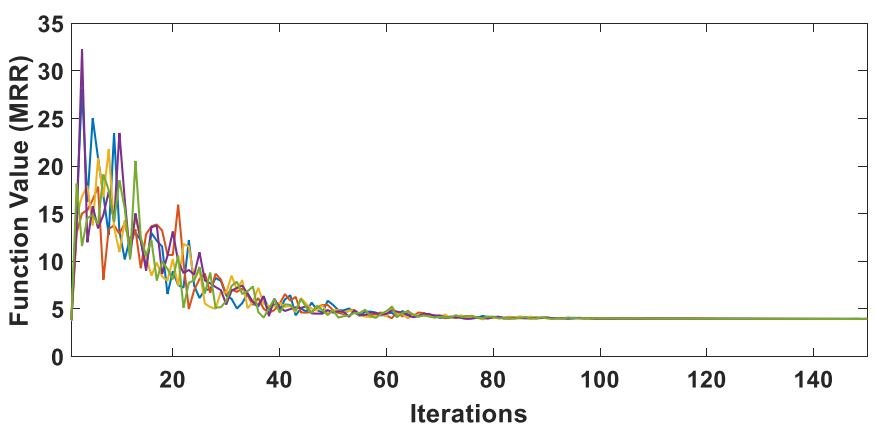

Fig. 5. Convergence plot for Ultrasonic Machining (USM) problem.

For USM problem, CI algorithm has obtained better solutions as compare to GA [34] and HS M [31] and precisely similar as compared, PSO and $\mathrm{AB} C$ [31]. The best, mean and worst reported solutions obtained form 30 trials using CI algorithm are $3.9375 \mathrm{~mm}^{3} / \mathrm{s}, 3.9373 \mathrm{~mm}^{3} / \mathrm{s}$ and $3.8391 \mathrm{~mm}^{3} / \mathrm{s}$ with standard deviation 0.0868 . The average function evaluations are 1209 and average computational time is $0.47 \mathrm{~s}$. The other computational details are illustrated in Table 5 . The convergence plot is presented in Figure 5.

\section{Result analysis and discussion}

The non-traditional single objective, multi variable nonlinear constrained AWJM problem and USM problem $[31,34]$ were successfully solved using constrained version of CI algorithm. CI algorithm was run for 30 times to

Table 4. Comparison of results for solving Ultrasonic Machining problem.

\begin{tabular}{lllllll} 
Techniques & \multicolumn{1}{c}{$A v$} & $f v$ & $d m$ & Cav & Fs & $\mathrm{MRR} \frac{\mathrm{mm}^{3}}{\mathrm{~s}}$ \\
\hline GA [34] & 0.0263 & $39,333.9$ & 0.133 & 0.479 & 10.8 & 3.553 \\
ABC [31] & 0.0167 & 40,000 & 0.15 & 0.5 & 16.4 & 3.941 \\
HS_M [31] & 0.0582 & 40,000 & 0.15 & 0.5 & 4.5 & 3.870 \\
PSO [31] & 0.06 & 40,000 & 0.15 & 0.5 & 4.5 & 3.950 \\
\hline
\end{tabular}

Table 5. Solutions of CI algorithm.

\begin{tabular}{lllllll}
\hline Problems & $\begin{array}{l}\text { CIBest } \\
\text { Meanworst }\end{array}$ & $\begin{array}{l}\text { Standard } \\
\text { deviation }\end{array}$ & $\begin{array}{l}\text { Average number } \\
\text { of function } \\
\text { evaluations }\end{array}$ & $\begin{array}{l}\text { Average } \\
\text { Computational } \\
\text { time (sec) }\end{array}$ & $\begin{array}{l}\text { \% improvement } \\
\text { over the best } \\
\text { reported solution }\end{array}$ & Parameters \\
\hline AWJM & $\begin{array}{l}97.7921 \\
93.1370\end{array}$ & 1.4686 & 591 & 0.81 & 8.0098 & $C=5$ \\
& 90.9787 & & & & & $R=0.95$ \\
USM & 3.9375 & 0.0868 & 1209 & 0.47 & $0.3164^{*}$ & $C=5$ \\
& 3.9373 & & & & & $R=0.95$ \\
\hline
\end{tabular}


analyse the effectiveness and robustness of algorithm. The statistical results for both the problems are presented in Table 5. It represents the best, mean and worst function values, average function evaluations, average computational time, closeness to the reported solution and the set of parameters required to run the CI algorithm. The constrained involved with these problems were handled using static penalty function approach. For maximization of MRR, AWJM problem was previously solved using GA [34], PSO and ABC [31]; however, CI obtained $8.0098 \%$ better solutions about with less computational time and function evaluations (refer Tab. 5). For USM problem, CI solution is $0.3164 \%$ worse than PSO [31]; whereas, HS_M [31] and GA [34] were slightly worst as compare to ${ }^{-} \mathrm{CI}$ algorithm. The probabilistic roulette wheel approach provided the possible choices to follow the best candidate in the cohort which assist the algorithm to escape the solution from local minima. Furthermore, the CI algorithm is depended on two parameter such as number of candidates $C$ and sampling space reduction factor $R$ which need to be tuned to obtain the better convergence within less computational cost.

\section{Conclusion}

The AWJM and USM problems are complex in nature and may not be able to solve using traditional gradient based optimization techniques. In this paper, a stochastic based CI algorithm is successfully applied to solve AWJM and USM problems for maximization of MRR. These problems are associated with linear and nonlinear constraints, a penalty function approach is used to handle the constraints. The solutions obtained from CI algorithm are successfully validated and obtained better solutions as comparing with GA, PSO, $\mathrm{ABC}$ and $\mathrm{HA} \mathrm{M}$. CI is incorporated with roulette wheel approach which make available to follow the best possible choices which helps the CI algorithm to obtained the better solution. In the near future CI algorithm can be applied for solving similar real-world application form advance manufacturing domain problem, complex healthcare and logistic domain problem.

\section{Abbreviations}

$\begin{array}{ll}\text { ABC } & \text { Artificial Bee Colony Algorithm } \\ \text { AMP } & \text { Advance Machining Process } \\ \text { ANFIS } & \text { Adaptive Neuro Fuzzy Inference System } \\ \text { AWJM } & \text { Abrasive Water Jet Machining } \\ \text { BSA } & \text { Binary Search Algorithm } \\ \text { CI } & \text { Cohort Intelligent Algorithm } \\ \text { CLPSO } & \text { Comprehensive Learning ParticleSwarm Optimizer } \\ \text { COA } & \text { Cuckoo Optimization Algorithm } \\ \text { CSO } & \text { Chicken Swarm Optimization } \\ \text { FW } & \text { Fireworks Algorithm. } \\ \text { GA } & \text { Genetic Algorithm } \\ \text { GSO } & \text { Gravitational Search Optimization } \\ \text { HH } & \text { Hoopoe Heuristic } \\ \text { HS M } & \text { Modified Harmony Search } \\ \text { ICA } & \text { Independent Component Analysis }\end{array}$

MRR Material Removal Rate

PSO Particle Swarm Optimization

SA Simulated Annealing

SFL Shuffled Frog Leaping

SPF Static Penalty Function approach

TLBO Teaching Learning Based Optimization

USM Ultrasonic Machining

WJM Water Jet Machining

\section{Nomenclature}

$h_{c} \quad$ Indentation depth due to cutting wear

$h_{d} \quad$ Indentation depth due to deformation wear

$\sigma_{f w} \quad$ Flow stress of the work material

$A_{t} \quad$ Cutting tool Cross-sectional area

$A_{v} \quad$ Amplitude of vibration

$C_{a v} \quad$ Abrasive grains volumetric concentration in slurry

$F_{s} \quad$ Static feed force

$M_{W} \quad$ Water Mass flow rate

$M_{a} \quad$ Abrasives Mass flow rate

$P_{W} \quad$ Water jet pressure at the nozzle exit

$R a_{\max }$ Allowable surface roughness value

$d_{\text {awn }}$ Abrasive-water jet nozzle Diameter

$d_{m} \quad$ Mean diameter of abrasive grains

$f_{n} \quad$ Nozzle traverse or feed rate

$f_{v} \quad$ Frequency of vibration

$r_{m} \quad$ Mean radius of abrasive particles

$v_{a} \quad$ Velocity of abrasive particles

$\alpha_{0} \quad$ Angle of impact at which erosion peaks

$\alpha_{t} \quad$ Angle of impact at top of cutting surface

$\rho_{a} \quad$ Abrasive particles Density

$\rho_{w} \quad$ Density of work material

$\sigma_{f t} \quad$ Abrasive particles Flow stress

$\alpha \quad$ Angle of impact

\section{References}

1. R.G. Gould, The LASER, light amplification by stimulated emission of radiation, in the Ann Arbor Conference on Optical Pumping, the University of Michigan 15, 92 (1959

2. N. Taylor, LASER: The inventor, the Nobel laureate, and the thirty-year patent war (Simon and Schuster, 2002)

3. E.C. Jameson, Electrical Discharge Machining (Society of Manufacturing Engineers, 2001)

4. K.P. Rajurkar, D. Zhu, J.A. McGeough, J. Kozak, A. De Silva, New developments in electro-chemical machining, CIRP Ann. 48, 567-579 (1999)

5. M. Martins, R. Godina, C. Pimentel, F.J.G. Silva, J.C. Matias, A practical study of the application of SMED to electron-beam machining in automotive industry, Proc. Manufactur. 17, 647-654 (2018)

6. P.L. Guzzo, A.H. Shinohara, A.A. Raslan, A comparative study on ultrasonic machining of hard and brittle materials, J. Br. Soc. Mech. Sci. Eng. 26, 56-61 (2004)

7. L. Alting, Manufacturing Engineering Processes (Marcel Dekker Inc., New York, 1982)

8. Z. Jurkovic, M. Perinic, S. Maricic, M. Sekulic, V. Mandic, Application of modelling and optimization methods in abrasive water jet machining, J. Trends Dev. Mach. Assoc. Technol. 16, 59-62 (2012) 
9. N. Kumar, M. Shukla, Finite element analysis of multiparticle impact on erosion in abrasive water jet machining of titanium alloy, J. Comput. Appl. Math. 236, 4600-4610 (2012)

10. H. Li, J. Wang, An experimental study of abrasive waterjet machining of Ti-6Al-4V, Int. J. Adv. Manufactur. Technol. 81, 361-369 (2015)

11. R.V. Rao, P.J. Pawar, J.P. Davim, Parameter optimization of ultrasonic machining process using nontraditional optimization algorithms, Mater. Manufactur. Process. 25, 1120 1130 (2010)

12. D. Goswami, S. Chakraborty, Parametric optimization of ultrasonic machining process using gravitational search and fireworks algorithms, Ain Shams Eng. J. 6, 315-331 (2015)

13. R. Teimouri, H. Baseri, R. Moharami, Multi-responses optimization of ultrasonic machining process, J. Intell. Manufactur. 26, 745-753 (2015)

14. B. Acherjee, D. Maity, A.S. Kuar, Ultrasonic machining process optimization by cuckoo search and chicken swarm optimization algorithms, Int. J. Appl. Metaheur. Comput. 11, 1-26 (2020)

15. D.H. Wolpert, K. Tumer, An Introduction to Collective Intelligence, Technical Report, NASA ARC-IC-99-63, NASA Ames Research Center, 1999

16. M.Y. Cheng, D. Prayogo, Symbiotic organisms search: a new metaheuristic optimization algorithm, Comput. Struct. 139, 98-112 (2014)

17. R.V. Rao, Advanced modeling and optimization of manufacturing processes (International Research and Development, Springer, 2011)

18. A.J. Kulkarni, I.P. Durugkar, M. Kumar, Cohort intelligence: a self-supervised learning behavior, in 2013 IEEE International Conference on Systems, Man, and Cybernetics (2013) pp. 1396-1400

19. A.J. Kulkarni, H. Shabir, Solving 0-1 knapsack problem using cohort intelligence algorithm, Int. J. Mach. Learn. Cybern. 7, 427-441 (2016)

20. A.J. Kulkarni, M.F. Baki, B.A. Chaouch, Application of the cohort-intelligence optimization method to three selected combinatorial optimization problems, Eur. J. Oper. Res. 250, 427-447 (2016)

21. A.J. Kulkarni, G. Krishnasamy, A. Abraham, Cohort intelligence: a socio-inspired optimization method (Springer International Publishing, Switzerland, 2017), p. 114

22. M. Aladeemy, S. Tutun, M.T. Khasawneh, A new hybrid approach for feature selection and support vector machine model selection based on self-adaptive cohort intelligence, Exp. Syst. Appl. 88, 118-131 (2017)

23. O. Kulkarni, N. Kulkarni, A.J. Kulkarni, G. Kakandikar, Constrained cohort intelligence using static and dynamic penalty function approach for mechanical components design, Int. J. Parallel Emerg. Distrib. Syst. 33, 570-588 (2018)

24. A.S. Shastri, A. Nargundkar, A.J. Kulkarni, K.K. Sharma, Multi-Cohort intelligence algorithm for solving advanced manufacturing process problems, Neural Comput. Appl. 32, 15055-15075 (2020)

25. V. Gulia, A. Nargundkar, Optimization of process parameters of abrasive water jet machining using variations of cohort intelligence (CI), in Applications of Artificial Intelligence Techniques in Engineering (2019), pp. 467-474

26. I.R. Kale, A.J. Kulkarni, Cohort intelligence algorithm for discrete and mixed variable engineering problems, Int. J. Parallel Emerg. Distrib. Syst. 33, 627-662 (2018)

27. I.R. Kale, A.J. Kulkarni, S.C. Satapathy, A socio-based cohort intelligence algorithm for engineering problems. Socio-cultural inspired metaheuristics, Stud. Comput. Intell. 828, 121-135 (2019)

28. I.R. Kale, A.J. Kulkarni, Cohort intelligence with selfadaptive penalty function approach hybridized with colliding bodies optimization algorithm for discrete and mixed variable constrained problems, Complex Intell. Syst. 7, 1565-1596 (2021)

29. D.E. Goldberg, Genetic Algorithms in Search, Optimization and Machine Learning (Addison-Wesley, 1989)

30. V.K. Jain, Advanced Machining Processes (Allied Publishers, 2009)

31. R.V. Rao, Advanced Modeling and Optimization of Manufacturing Processes (International Research and Development, Springer, 2010)

32. J. Wang, Abrasive Waterjet Machining of Engineering Materials (Trans Tech Publications Ltd, Switzerland, Zurich, 2003)

33. M. Hashish, A modeling study of metal cutting with abrasive water jets, J. Eng. Mater. Technol. 106, 88-100 (1984)

34. N.K. Jain, V.K. Jain, K. Deb, Optimization of process parameters of mechanical type advanced machining processes using genetic algorithms, Int. J. Mach. Tools Manufact. 47, 900-919 (2007)

35. A.V. Mitrofanov, V.I. Babitsky, V.V. Silberschmidt, Finite element analysis of ultrasonically assisted turning of Inconel 718, J. Mater. Process. Technol. 153, 233-239 (2004) 